\title{
Atypical Presentation of Spontaneous Coronary Artery Dissection
}

\author{
Chidi D. Okoroafor ${ }^{1}$, Abeera Akram ${ }^{1}$, Annie Ong ${ }^{1}$ \\ 1. Internal Medicine, Saint Mary's Hospital, Waterbury, USA
}

Corresponding author: Chidi D. Okoroafor, chidi.okoroafor@trinityhealthofne.org

\begin{abstract}
Spontaneous coronary artery dissection (SCAD) is a rare cause of acute coronary syndrome, more common in young women without risk factors for, or a history of, coronary artery disease and usually occurs in the peripartum period. There are two types of SCAD: atherosclerotic and nonatherosclerotic coronary artery dissection. Management options include conservative management, percutaneous coronary intervention, or surgical intervention, depending on the presentation and extent of the dissection. We present reports of two cases of SCAD (one man and one woman) presenting to the emergency department of our community hospital in February 2020 with nonspecific symptoms.
\end{abstract}

Categories: Cardiology, Internal Medicine

Keywords: atypical spontaneous coronary artery dissection, coronary artery dissection, spontaneous coronary dissection, : acute coronary syndrome

\section{Introduction}

Acute coronary syndrome (ACS) is one of the leading causes of presentation to the emergency department (ED) for chest pain. Many patients with ACS at presentation may have myocardial infarction. . Spontaneous coronary artery dissection (SCAD) is a rare cause of ACS, and chest pain is usually the most common presentation [1]. We present the cases of two patients (one man, one woman) with SCAD who presented to the ED of our community hospital in February 2020 with nonspecific symptoms.

\section{Case Presentation}

\section{Case 1}

A 48-year-old obese man who is a current smoker with a history of chronic obstructive pulmonary disease (COPD) presented with shortness of breath and productive cough to the ED. At presentation, he was afebrile, tachycardic (heart rate, 120 beats/minute [bpm]), tachypneic (respiratory rate, 35 breaths/minute), and hypoxic (oxygen saturation, $79 \%$ on room air). Jugular venous distention was difficult to appreciate. He had bilateral wheezing throughout the lung fields and $1+$ pitting edema in both lower limbs. He was put on $5 \mathrm{~L}$ oxygen via nasal cannula, given intravenous (IV) steroids, albuterol nebulization, IV furosemide, and doxycycline. His arterial blood gas showed $\mathrm{pH}$ of 7.31, partial pressure of $\mathrm{CO}_{2}$ of $59 \mathrm{mmHg}$, partial pressure of $\mathrm{O}_{2}$ of $57 \mathrm{mmHg}$, and a bicarbonate of $26 \mathrm{mmol} / \mathrm{L}$. He was started on bilevel positive airway pressure (BiPAP) at $50 \%$ oxygen with an inspiratory positive airway pressure of 12 and an expiratory positive airway pressure of 5 in the setting of severe hypercapnic hypoxic respiratory failure secondary to COPD

He was admitted to the telemetry monitoring unit and continued BiPAP support with ipratropium/albuterol combination nebulization. The patient denied chest pain, but his electrocardiogram (ECG) showed a newonset left bundle branch block (Figure 1) as compared to his ECG from six weeks prior (Figure 2). His troponin levels were $0.04 \mathrm{ng} / \mathrm{ml}$ at presentation, $0.04 \mathrm{ng} / \mathrm{ml} 2$ hours later and $<0.03 \mathrm{ng} / \mathrm{ml}$ after 12 hours. 


\section{Cureus}

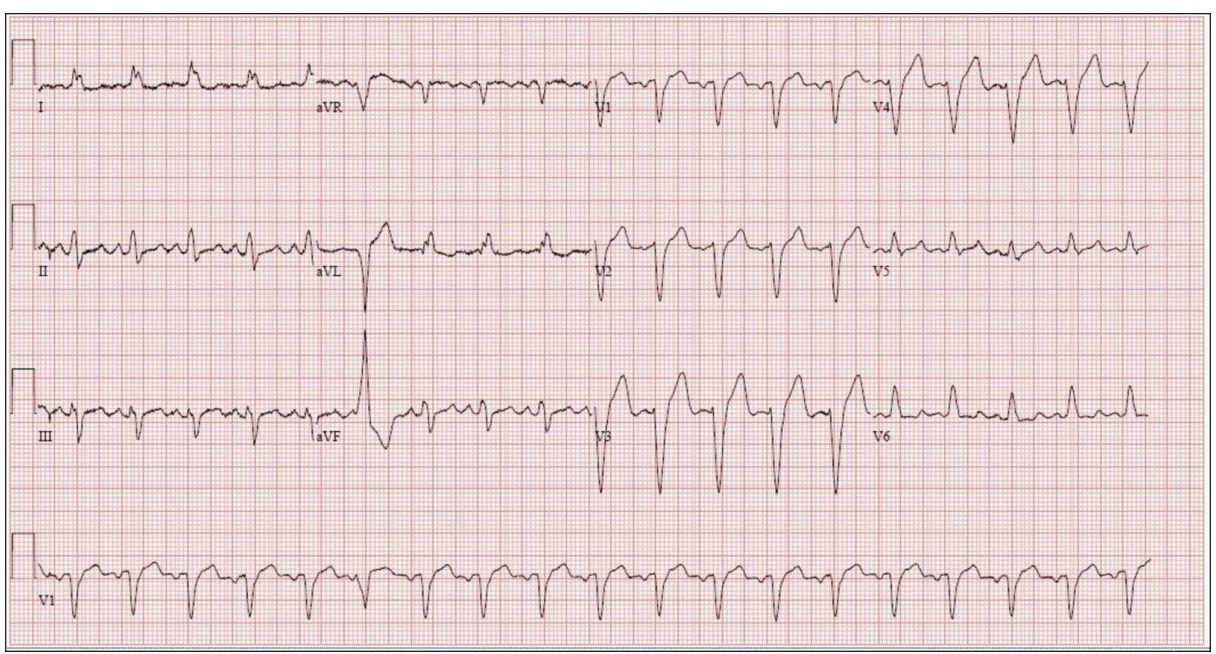

FIGURE 1: Case 1 electrocardiogram at presentation showing a normal sinus rhythm with a new left bundle branch block

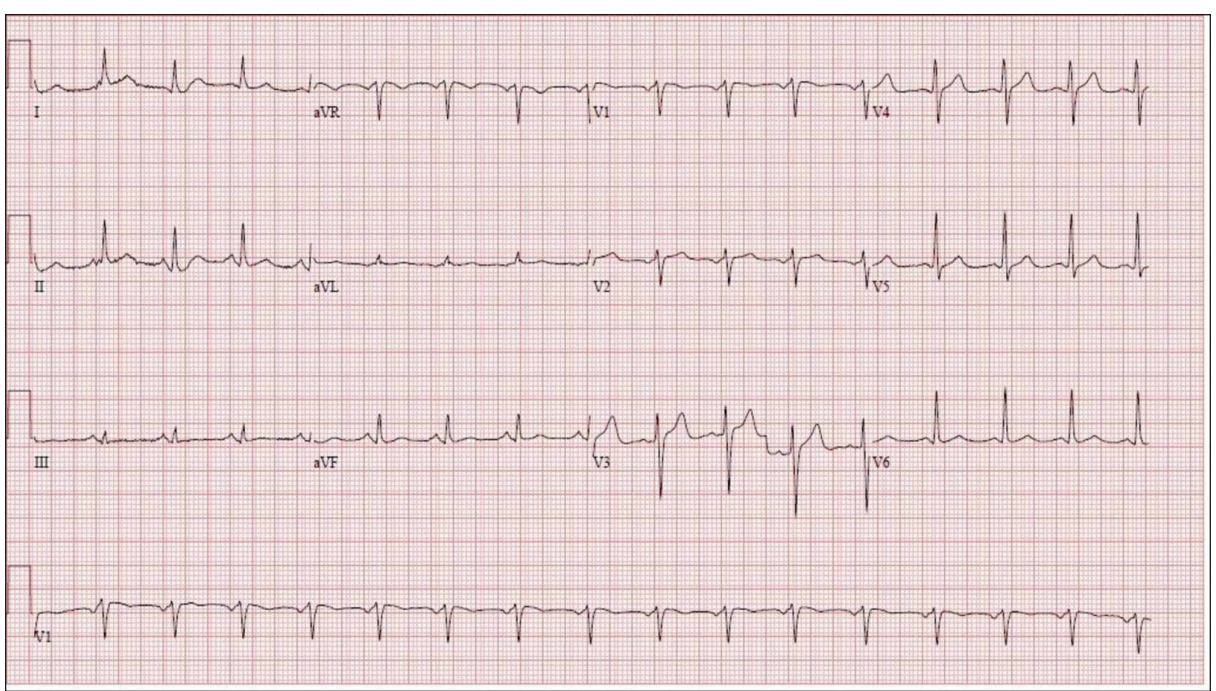

FIGURE 2: Case 1 baseline electrocardiogram six weeks prior to presentation showing a normal sinus rhythm.

A transthoracic echocardiogram showed severe left ventricular dysfunction with global hypokinesis and an ejection fraction (EF) of 30\% to 35\%. Cardiac catheterization showed nonischemic cardiomyopathy and SCAD of the first obtuse marginal artery, which is a type 1 dissection (Figure 3). He had thrombolysis in myocardial infarction (TIMI) score of 3 but no chest pain. Given the patient's history of poor follow-up with outpatient care, the patient was managed medically and percutaneous coronary intervention (PCI) was not performed. He was started on aspirin, clopidogrel, sacubitril/valsartan, furosemide, and atorvastatin. He was advised to have close follow-up with a cardiologist. 


\section{Cureus}

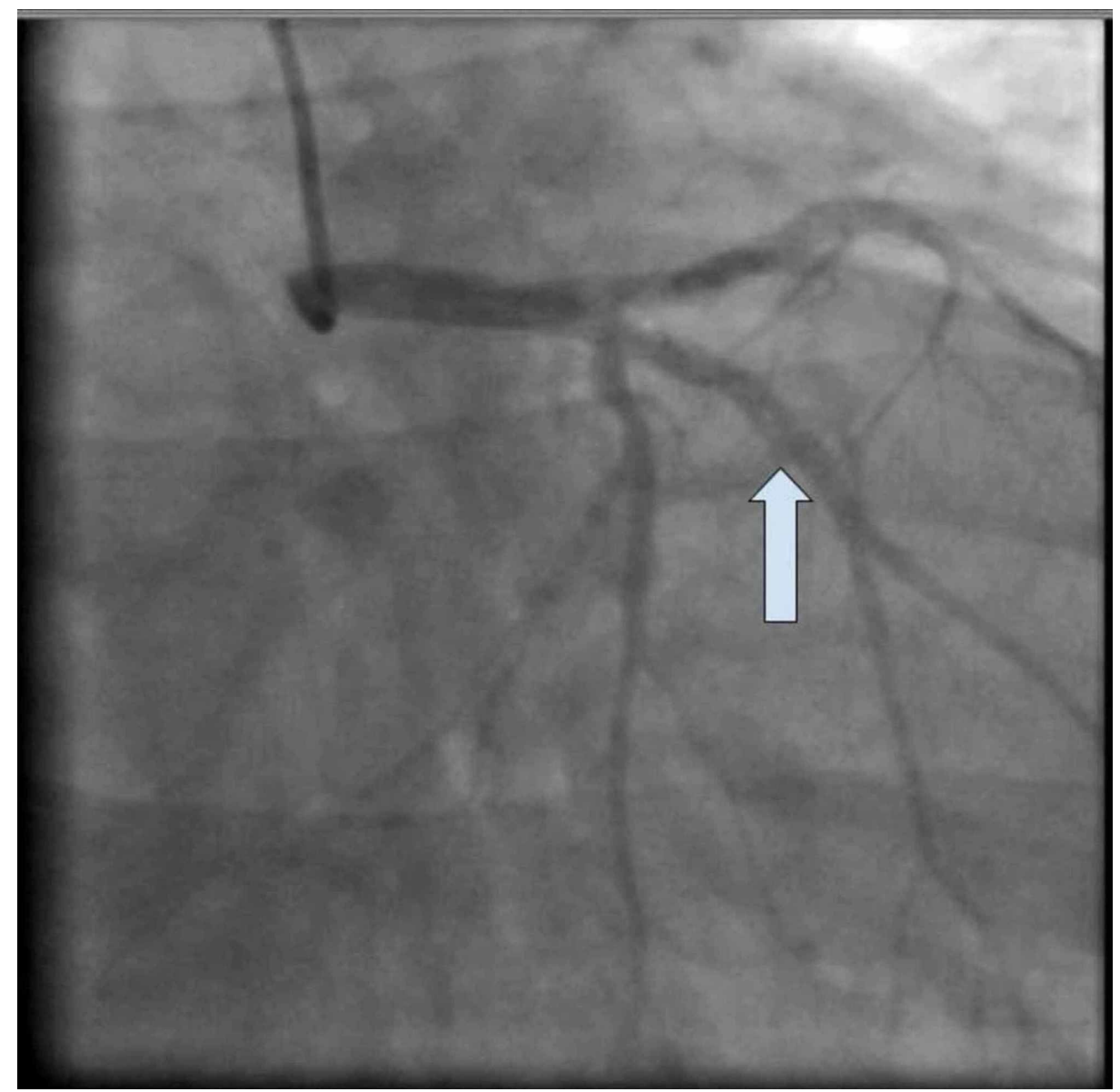

FIGURE 3: Case 1: Type 1 dissection of the first obtuse marginal artery.

\section{Case 2}

A nonsmoking, obese, active 38-year-old woman presented to the ED with recurrent episodes of heartburn following a meal. On the day of presentation, her heartburn was persistent, more severe, and was not relieved by calcium carbonate tablets as the symptom normally did. At presentation, she was afebrile, with a heart rate of $71 \mathrm{bpm}$, a respiratory rate of 16 breaths per minute, a blood pressure of 130/78 $\mathrm{mmHg}$, and oxygen saturation of $99 \%$ on room air. She had no signs of poor perfusion or volume overload. Her ECG was normal (Figure 4); however, her troponin was elevated at $0.20 \mathrm{ng} / \mathrm{ml}$ and trended up to a peak of $2.03 \mathrm{ng} / \mathrm{ml}$. Her transthoracic echocardiography was only significant for mild concentric left ventricular hypertrophy with an $\mathrm{EF}$ of $55 \%$ to $65 \%$.

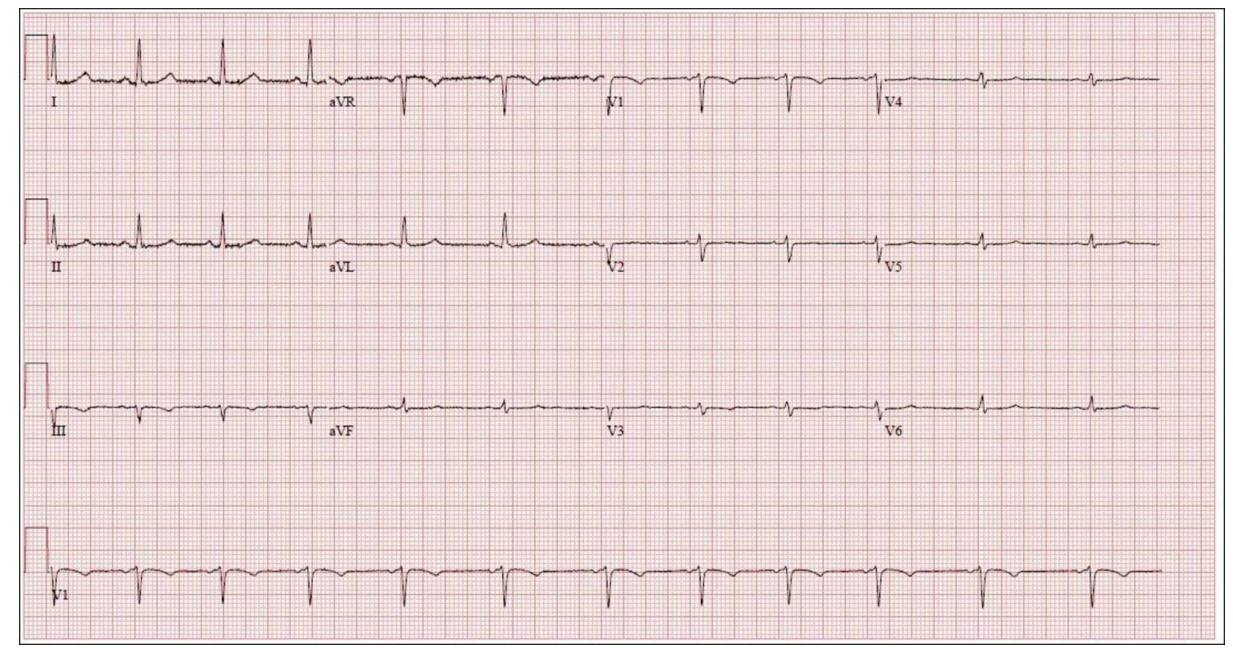

FIGURE 4: Case 2 electrocardiogram at presentation showing a normal 


\section{Cureus}

sinus rhythm. It also meets criteria for low voltage in the precordial leads but that is not the scope of this presentation.

Cardiology was consulted because of the patient's troponin elevation in the setting of atypical chest pain. Cardiac catheterization revealed SCAD of the distal left anterior descending artery, which is a type 2 dissection (Figure 5). Given her TIMI-3 flow and absence of typical chest pain, we decided to treat her medically. She was started on metoprolol, aspirin, and atorvastatin. Given her young age and family history of early death due to brain aneurysm, we conducted further workup for vasculitis, autosomal dominant polycystic kidney disease, and fibromuscular dysplasia (FMD) via bilateral renal ultrasound, computed tomography angiography of the chest and abdomen, brain magnetic resonance imaging and magnetic resonance angiography, and tests for anti-neutrophilic cytoplasmic autoantibody-associated vasculitis, the results of which were all unremarkable. She was discharged on metoprolol, aspirin, and atorvastatin with a scheduled follow-up evaluation with a cardiologist.

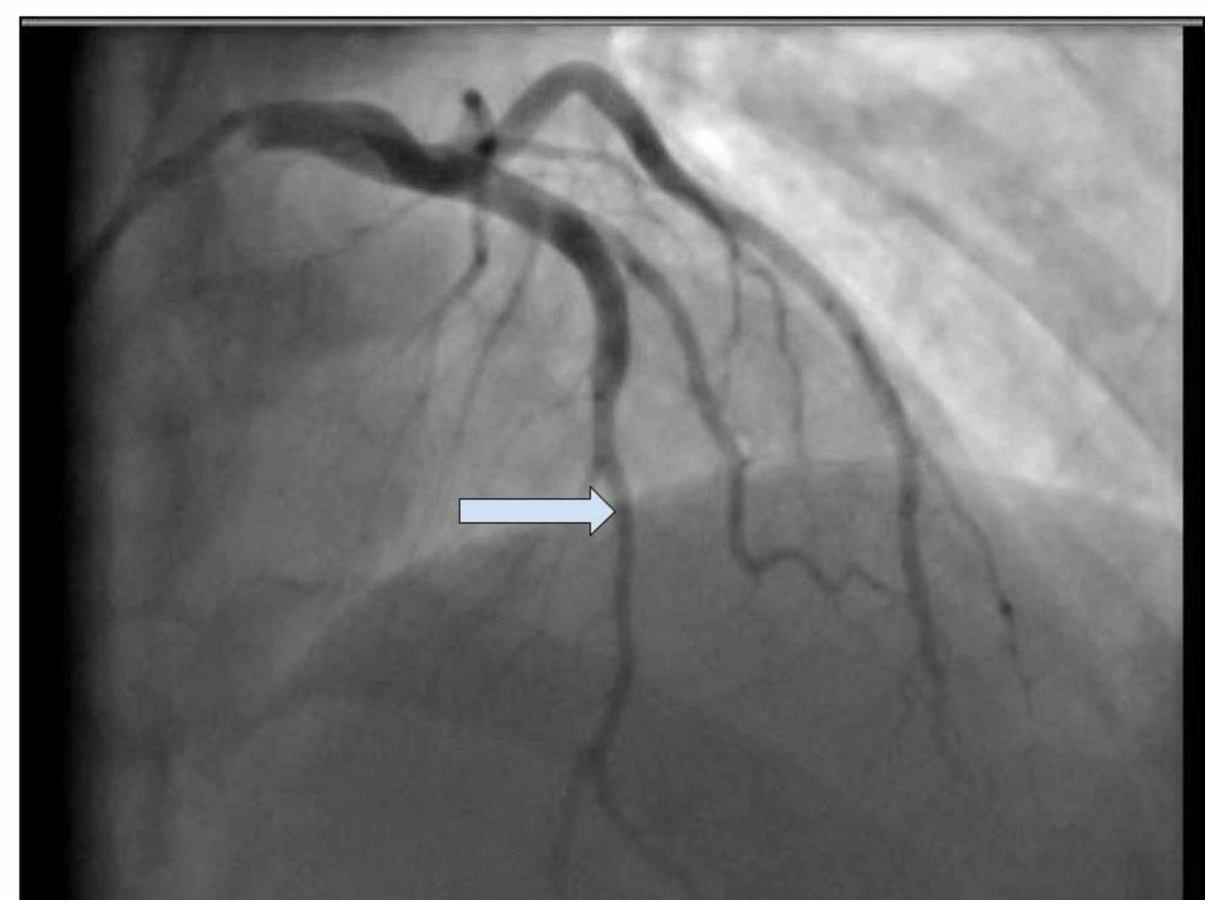

FIGURE 5: Case 2: Type 2 dissection of the distal left anterior descending.

\section{Discussion}

SCAD is a nontraumatic and noniatrogenic separation of the coronary arterial wall and is a rare cause of acute myocardial infarction and ACS, accounting for $0.1 \%$ to $0.4 \%$ of ACS cases [1]. SCAD is more common in young women in the peripartum period, usually without cardiovascular risk factors; in men of any age, SCAD accounts for only $10 \%$ to $15 \%$ of cases of ACS. Also, for men, atherosclerotic SCAD is more common than nonatherosclerotic SCAD [2]. There is some association of SCAD in men related to exertional stress, anxiety, and emotional stress [3].

Weakening of the arterial wall due to hormonal changes, cystic medial necrosis, and proteolytic enzymes from periadventitial eosinophils in susceptible patients can lead to an underlying vulnerable coronary wall [4]. Underlying factors and conditions that could potentially predispose a patient to SCAD are FMD, postpartum status, multiparity more than four births, connective tissue disorders, systemic inflammatory conditions, and hormonal therapy [5]. Stressors such as intense exercise or emotional stress, labor and delivery, intense Valsalva-type activities, and recreational drug use can precipitate SCAD [3]. SCAD can present with a variety of features including arrhythmias, unstable angina, acute myocardial infarction, cardiogenic shock, pericardial effusion and tamponade, and even sudden cardiac death [3,6-8]. 
Atherosclerotic SCAD is a mechanistically distinct variant of SCAD and is typically limited in extent by medial atrophy and scarring associated with atherosclerotic cardiovascular disease. Unlike atherosclerotic SCAD, nonatherosclerotic SCAD can result in extensive dissection lengths, especially in the presence of arterial fragility from predisposing arteriopathies [9].

The exact mechanism of nonartherosclerotic SCAD is poorly understood. An intimal tear or bleeding of the vasa vasorum with intramedial hemorrhage has been proposed, with both processes leading to the creation of a false lumen [4]. Pressure-driven expansion of the false lumen induces axial propagation of the intramural hematoma, luminal encroachment, and ultimately myocardial ischemia and infarction.

Chest pain is the most common symptom presentation in $>96 \%$ of cases; less common symptoms include arm pain, neck pain, nausea or vomiting, diaphoresis, dyspnea, and back pain $[1,6,10]$. In most patients, in the absence of prior trauma, the diagnosis of SCAD is made at the time of coronary angiography.

The criteria for the angiographic definition of SCAD include the presence of a noniatrogenic dissection plane in the absence of coronary atherosclerosis, with typical changes of radiolucent intimal flap and contrast staining. An angiographic series has shown that such stereotypical changes were seen in only less than $30 \%$ of nonatherosclerotic SCAD cases [5,11]. The majority of SCAD had long and diffuse narrowing on angiography due to intramural hematoma, and this appearance was frequently unrecognized on angiography leading to underdiagnosis of this condition.

Type 1 dissection: Pathognomonic contrast dye staining of arterial wall with multiple radiolucent lumen, with or without the presence of dye hang-up or slow contrast clearing.

Type 2 dissection: Diffuse long and smooth stenosis that can vary in severity from mild stenosis to complete occlusion.

Type 3 dissection: Mimics atherosclerosis with focal or tubular stenosis and requiring optical coherence tomography (OCT) or intravascular ultrasound (IVUS) to differentiate the cause.

In patients for whom the diagnosis is considered but not secured with coronary angiography, intracoronary imaging with OCT or IVUS may be helpful. With these imaging modalities, SCAD diagnosis is made with the presence of intramural hematoma and/or a double lumen. Alternatively, repeat coronary angiography may be pursued four to six weeks later to evaluate for spontaneous angiographic healing of the dissected segment, if the diagnosis is uncertain.

Irrespective of dissection type in most SCAD patients, conservative management with medical therapy is the preferred strategy as was the case with our patients. PCI can be used for patients needing emergent revascularization [3]. Other treatment options include coronary artery bypass grafting, fibrinolytic therapy (with or without subsequent PCI), mechanical hemodynamic support, and cardiac transplantation, the selection of which should be based on patient presentation and unique characteristics [5].

\section{Conclusions}

SCAD as a rare cause of ACS has been shown to be associated with various presentations. Some of which include anxiety, emotional stress, exertional stress, and intense Valsalva-type activities usually with associated chest pain. In most patients, conservative management with medical therapy is the preferred approach to management. Our case series highlights the need to also consider the diagnosis of SCAD as a cause of ACS in young patients presenting to the ED with nonspecific symptoms, such as shortness of breath and heartburn, and not just those with the more typical presentation of chest pain.

\section{Additional Information \\ Disclosures}

Human subjects: Consent was obtained by all participants in this study. Conflicts of interest: In compliance with the ICMJE uniform disclosure form, all authors declare the following: Payment/services info: All authors have declared that no financial support was received from any organization for the submitted work. Financial relationships: All authors have declared that they have no financial relationships at present or within the previous three years with any organizations that might have an interest in the submitted work. Other relationships: All authors have declared that there are no other relationships or activities that could appear to have influenced the submitted work.

\section{References}

1. Mortensen KH, Thuesen L, Kristensen IB, Christiansen EH: Spontaneous coronary artery dissection: a Western Denmark Heart Registry study. Catheter Cardiovasc Interv. 2009, 74:710-717. 10.1002/ccd.22115

2. Alfonso F, Paulo M, Lennie V, et al.: Spontaneous coronary artery dissection: long-term follow-up of a large series of patients prospectively managed with a “conservative” therapeutic strategy. JACC Cardiovasc 


\section{Cureus}

Interv. 2012, 5:1062-1070. 10.1016/j.jcin.2012.06.014

3. Thomas BN, Aslam S, Cullen J, Anantharaman R: Spontaneous coronary artery dissection in men presenting with acute coronary syndrome, successfully managed by intravascular ultrasound-guided percutaneous coronary intervention. BMJ Case Rep. 2014, 2014:bcr2013009169. 10.1136/bcr-2013-009169

4. Alfonso F: Spontaneous coronary artery dissection: new insights from the tip of the iceberg? . Circulation. 2012, 126:667-670. 10.1161/CIRCULATIONAHA.112.122093

5. Saw J, Aymong E, Sedlak T, et al.: Spontaneous coronary artery dissection: association with predisposing arteriopathies and precipitating stressors and cardiovascular outcomes. Circ Cardiovasc Interv. 2014, 7:645655. 10.1161/CIRCINTERVENTIONS.114.001760

6. Tweet MS, Hayes SN, Pitta SR, et al.: Clinical features, management, and prognosis of spontaneous coronary artery dissection. Circulation. 2012, 126:579-588. 10.1161/CIRCULATIONAHA.112.105718

7. Saw J, Starovoytov A, Humphries K, et al.: Canadian spontaneous coronary artery dissection cohort study: in-hospital and 30-day outcomes. Eur Heart J. 2019, 40:1188-1197. 10.1093/eurheartj/ehz007

8. Lobo AS, Cantu SM, Sharkey SW, et al.: Revascularization in patients with spontaneous coronary artery dissection and ST-segment elevation myocardial infarction. J Am Coll Cardiol. 2019, 74:1290-1300. 10.1016/j.jacc.2019.06.065

9. Saw J, Mancini GB, Humphries K, Fung A, Boone R, Starovoytov A, Aymong E: Angiographic appearance of spontaneous coronary artery dissection with intramural hematoma proven on intracoronary imaging. Catheter Cardiovasc Interv. 2016, 87:E54-E61. 10.1002/ccd.26022

10. Luong C, Starovoytov A, Heydari M, Sedlak T, Aymong E, Saw J: Clinical presentation of patients with spontaneous coronary artery dissection. Catheter Cardiovasc Interv. 2017, 89:1149-1154. 10.1002/ccd.26977

11. Saw J, Humphries K, Aymong E, Sedlak T, Prakash R, Starovoytov A, Mancini GBJ: Spontaneous coronary artery dissection: clinical outcomes and risk of recurrence. J Am Coll Cardiol. 2017, 70:1148-1158. 10.1016/j.jacc.2017.06.053 